\title{
Nonlinear system identification for autonomous systems via functional equations methods
}

\author{
Alberto Padoan and Alessandro Astolfi
}

\begin{abstract}
The problem of identifying an autonomous nonlinear systems, that is, the problem of finding a state-space description of a given sequence generated by sampling the output of an unknown nonlinear system without input, is studied. A theoretical framework which combines the use of functional equations with realization-theoretic techniques is developed and used to solve the problem.
\end{abstract}

\section{INTRODUCTION}

The identification problem for linear dynamical systems has been solved both in a deterministic and a stochastic framework [1-8]. Prediction error methods and subspace identification methods broadly constitute the classical approaches to linear system identification $[1,2,5,7,8]$. The former revolves around the minimization of a prediction error criterion and is widely recognized as the state of the art for the identification of (single-input single-output) linear systems [1,2]. The latter is coordinate-free and based on geometric arguments which draw upon linear realization theory $[5,7,8]$.

The identification of nonlinear dynamical systems is more complex and, for many aspects, is still an open problem: only ad hoc methods or partial solutions for specifica classes of systems are available in literature. The proposed approaches make use of tools and algorithms originated in the most disparate areas of research, such as statistics, numerical analysis and control theory [2,9-13].

The adoption of a statistical approach to the identification of state-space nonlinear systems has been discussed, for example, in [14-16]. The extension of several linear identification methods to Hammerstein-Wiener systems has been discussed in [17-22]. The applicability of subspace identification methods to nonlinear systems has been studied, e.g., in [23-25]. A dedicated framework for discrete-time linear parameter-varying systems and for discrete-time bilinear state-space systems has been developed in [26]. Recently, subspace identification methods have been implemented in some applications, e.g., in the modelling of vibrating structures $[27,28]$. Further detail and an in-depth discussion on the latest developments in nonlinear systems identification can be found in the survey [13].

A. Padoan is with the Department of Electrical and Electronic Engineering, Imperial College London, London SW7 2AZ, UK. (Email: alberto.padoan130imperial.ac.uk). A. Astolfi is with the Department of Electrical and Electronic Engineering, Imperial College London, London SW7 2AZ, UK and with the Dipartimento di Ingegneria Civile e Ingegneria Informatica, Università di Roma "Tor Vergata", Via del Politecnico 1, 00133, Rome, Italy. (Email: a.astolfieimperial.ac.uk).
In the field of chaos theory, a completely different perspective has been taken by nonlinear time series analysts [29-34]. Building on the pioneering works [35] and [36], properties of nonlinear dynamical systems underlying measured time series have been studied by means of embedding techniques. The applicability of embedding methods, however, has been criticized by some authors (see, e.g., [37]). The reader may consult [12] for further detail on embedding methods.

Goal of this work is to propose a geometric framework for the solution of the identification problem for autonomous nonlinear systems based on the differential geometric approach to nonlinear systems $[38,39]$ and on the solution of the Schröder equation [40-42]: a functional equations that traces its roots to the linearization of nonlinear dynamical systems [43]. The exposition is inspired and motivated by ideas drawn from nonlinear realization theory and, with the exception of minor modifications, proceeds along the same lines as in [38]. To the best of the authors' knowledge, this is the first work where the Schröder equation is used for the purpose of identification. This paper is conceptual in nature and intended to provide a preliminary treatment of the subject. The authors are aware that important issues, such as identifiability and the effect of noise, are disregarded and will address these issues in future investigations.

The rest of the paper is organized as follows. Section II] formulates the identification problem for autonomous nonlinear systems. Section III contains a review of the necessary background material. Preliminary results are established in Section IV] Section $\mathrm{V}$ illustrates the main results. Finally, conclusions and future directions of research are outlined in Section VI.

Notation: Standard notation is used. $\mathbb{N}$ denotes the set of natural numbers (including 0 ). $\mathbb{R}, \mathbb{R}^{n}$ and $\mathbb{R}^{p \times m}$ denote the set of real numbers, of $n$-dimensional vectors with real entries and of $p \times m$-dimensional matrices with real entries, respectively. $\mathbb{R}_{+}$denotes the set of non-negative real numbers. $|x|$ denotes the standard Euclidean norm of the vector $x \in \mathbb{R}^{n} . \rho(A)$ denotes the spectral radius of the matrix $A \in \mathbb{R}^{n \times n} \cdot \varphi \circ \psi(\cdot)$ and $\varphi^{-1}(\cdot)$ denote the composition of the functions $\varphi(\cdot)$ and $\psi(\cdot)$ and the inverse function of $\varphi(\cdot)$, provided they exist. The iterates of a function $\varphi(\cdot)$ are defined recursively as $\varphi^{k+1}(\cdot)=\varphi^{k} \circ \varphi(\cdot)$, for all $k \in \mathbb{N}$, with $\varphi^{1}(\cdot)=\varphi(\cdot)$. No confusion should arise when superscripts are used for powers of matrices, since the meaning of the notation is clear from the context. The flow of a smooth vector field $f: \mathbb{R}^{n} \rightarrow \mathbb{R}^{n}$ passing through $x \in \mathbb{R}^{n}$ at time $t=0$ is denoted by $\phi_{t}^{f}(x)$, i.e. $t \mapsto \phi_{t}^{f}(x)$ is the integral curve of $f(\cdot)$ such that $\phi_{0}^{f}(x)=x$. 


\section{Problem Formulation}

Consider a continuous-time, autonomous, nonlinear system described by equations of the form

$$
\dot{x}=f(x), \quad y=h(x),
$$

in which $x(t) \in X$ and $y(t) \in \mathbb{R}^{p}$ denote the state and the output of the system at time $t$, respectively. The set $X$ is assumed to be a connected open subset of $\mathbb{R}^{n}$ such that $0 \in \operatorname{int} X$. The mappings $f: X \rightarrow X$ and $h: X \rightarrow \mathbb{R}^{p}$ are assumed to be analytip ${ }^{1}$ i.e. of class $C^{\omega}(X)$. Without loss of generality, the origin is assumed to be an equilibrium point for the system, i.e. $f(0)=0$ and $h(0)=0$. Finally, we make the following assumptions.

Assumption 1. The flow $\phi_{t}^{f}(\cdot)$ of the vector field $f(\cdot)$ is a global diffeomorphism ${ }^{2}$ on $X$.

Assumption 2. The equilibrium at the origin of system (1) is exponentially stable on $X$, i.e. there exist scalars $\eta_{1}>0$ and $\eta_{2}>0$ such that

$$
\left|\phi_{t}^{f}\left(x_{0}\right)\right| \leq \eta_{1} e^{-\eta_{2} t}\left|x_{0}\right|, \quad x_{0} \in X, \quad t \in \mathbb{R}_{+} .
$$

Remark 1. Assumption 2 implies the forward completeness of the vector field $f(\cdot)$ on $X$, i.e. the flow $\phi_{t}^{f}(\cdot)$ is defined for all $t \in \mathbb{R}_{+}$. Note also that, by Assumption 2 if system (11) is linear, i.e. it is described by equations of the form

$$
\dot{x}=A x, \quad y=C x,
$$

with $x(t) \in \mathbb{R}^{n}, y(t) \in \mathbb{R}^{p}, A \in \mathbb{R}^{n \times n}, C \in \mathbb{R}^{p \times n}$, then all the eigenvalues of the matrix

$$
A=\left.\frac{\partial f}{\partial x}\right|_{x=0}
$$

have negative real part (see [44, Theorem 3.13]).

The nonlinear identification problem for systems of the form (1) consists in finding

(i) the dimension $n>0$ of system (1), and

(ii) the mappings $f: X \rightarrow X$ and $h: X \rightarrow \mathbb{R}^{p}$,

from a given set $\left\{y\left(t_{k}\right)\right\}_{k=0}^{m}$ of observed output measurements, with $\left\{t_{k}\right\}_{k=0}^{m}$ a sequence of distinct real numbers.

Problem (i) is hard in general, except for special cases [2]. A simple way to circumvent this issue is to postulate the dimension of the state space of the system a priori and then evaluate the quality of each candidate solution according to some application-dependent criteria [2]. For this reason, we disregard the important issue of finding the dimension of the system, which is assumed to be given henceforth, and we concentrate on problem (ii). For further detail on problem (i) the reader may consult $[2,12,45]$, and references therein.

Since the primary interest of the paper is conceptual, we consider the ideal case in which $m=\infty$.

Remark 2. A global solution in $X$ to the identification problem posed above may not exist. While linear systems

\footnotetext{
1 Throughout the paper, if not otherwise stated, it is assumed that all functions and mappings are analytic.

${ }^{2} \mathrm{~A}$ smooth bijection $\tau(\cdot)$ is a diffeomorphism if its inverse map $\tau^{-1}(\cdot)$ is also smooth.
}

behave globally in the same way as they behave locally, it is not necessarily possible to establish global dynamical properties for most nonlinear systems. Without additional assumptions on the nonlinear system (1), the description of its dynamics may be determined only in a neighborhood of the (unknown) initial condition. Our analysis has therefore a local nature. In other words, we restrict our goal to finding the behaviour of the system mappings only in a sufficiently small open neighborhood containing the (unknown) initial condition.

Remark 3. There exist infinitely many nonlinear systems able to produce the output generated by system (1). More specifically, the system

$$
\dot{\chi}=f_{\tau}(\chi), \quad y=h_{\tau}(\chi),
$$

with $f_{\tau}(\chi)=\left[\frac{\partial \tau}{\partial x} f\right]_{x=\tau^{-1}(\chi)}, h_{\tau}(\chi)=[h]_{x=\tau^{-1}(\chi)}$, and $\tau(\cdot)$ a (local) diffeomorphism, has the same output behavior as the system (11). For this reason, the system mappings $f(\cdot)$ and $h(\cdot)$ cannot be uniquely identified from a set of output measurements without additional assumptions. To remove this shortcoming, any state-space realization of system (1) of the form (4) is considered acceptable. In other words, the solution of the posed identification problem is only determined up to a (local) diffeomorphism.

The main idea of this work is to reduce the identification problem for systems of the form (1) to the solution of the Schröder equation [40-42]

$$
\Psi \circ \varphi(x)=S \Psi(x), \quad x \in N,
$$

in the unknown function $\Psi(\cdot)$, in which

- $N$ is a neighborhood of the origin,

- $\varphi(\cdot)=\phi_{\delta}^{f}(\cdot)$,

- $S=e^{A \delta}$, with $A$ as in (3) and $\delta$ a positive real number, provided both sides of equation (5) are defined.

The solution of (5) allows to express the iterates $\varphi^{k}(\cdot)$ as

$$
\varphi^{k}(x)=\Psi^{-1}\left(S^{k} \Psi(x)\right), \quad x \in N, \quad k \in \mathbb{N},
$$

the flow $\phi_{t}^{f}(\cdot)$ as

$$
\phi_{t}^{f}(x)=\Psi^{-1}\left(S^{\frac{t}{\delta}} \Psi(x)\right), \quad x \in N, \quad t \in \mathbb{R}_{+},
$$

and the vector field $f(\cdot)$ as

$$
f(x)=\left.\frac{d}{d t} \phi_{t}^{f}(x)\right|_{t=0}, \quad x \in N .
$$

Borrowing a construction from nonlinear realization theory [38], one may manufacture a function $h: N \rightarrow \mathbb{R}^{p}$ compatible with the given output data, i.e. such that

$$
y\left(t_{k}\right)=h \circ \phi_{t_{k}}^{f}(x), \quad k \in \mathbb{N},
$$

for some $x \in N$, which determines a state-space description able to generate the prescribed output behaviour when initialized at some specific point of the state space, and thus solves the identification problem for systems of the form (1). 
Note that, under the stated assumptions, equation (5) has a unique local analytic solution in a neighborhood of the origin fulfilling the condition

$$
\left.\frac{\partial \Psi}{\partial x}\right|_{x=0}=I
$$

with $I$ the identity matrix, in virtue of [41, Theorem 8.2.1].

Note also that the restrictive assumption of knowing the function $\varphi(\cdot)$ can be relaxed. In particular, it suffices to know $\varphi(\cdot)$ up to a change of coordinates, as formalized by the following result.

Lemma 1. Let $\delta>0$ be a real number. If $\Psi(\cdot)$ solves (5), then

$$
\bar{\Psi}(\cdot)=T^{-1} \Psi \circ \tau(\cdot)
$$

solves $\bar{\Psi} \circ \bar{\varphi}(\chi)=\bar{S} \bar{\Psi}(\chi), \chi \in M$, with

- $\tau: M \rightarrow N$ a diffeomorphism of neighborhoods of the origin such that $\tau(0)=0$ and

$$
T=\left.\frac{\partial \tau}{\partial \chi}\right|_{\chi=0} ;
$$

- $\bar{\varphi}(\cdot)$ a function that is locally $\tau$-conjugate to $\phi_{\delta}^{f}(\cdot)$, i.e.

$$
\bar{\varphi}(x)=\tau^{-1} \circ \phi_{\delta}^{f} \circ \tau(\chi), \quad \chi \in M,
$$

- $\bar{S}=T S T^{-1}$.

Proof. The claim follows by direct computation, since

$$
\begin{aligned}
\bar{\Psi} \circ \bar{\varphi} & =\left(T^{-1} \Psi \circ \tau\right) \circ\left(\tau^{-1} \circ \varphi \circ \tau\right)=T^{-1}(\Psi \circ \varphi) \circ \tau \\
& =T^{-1}(S \Psi) \circ \tau=\left(T^{-1} S T\right)\left(T^{-1} \Psi \circ \tau\right)=\bar{S} \bar{\Psi},
\end{aligned}
$$

in which, for the sake of clarity, the arguments of the functions have been omitted.

\section{BACKGROUND MATERIAL}

This section contains a brief review of elementary notions concerning formal power series [46,47], nonlinear control theory [38,39], and sampled-data control theory [48] needed in the following sections.

\section{A. Formal power series and sequences}

A formal power series in one indeterminate with coefficients in $\mathbb{R}^{p}$ is a mapping of the form $s: \mathbb{N} \rightarrow \mathbb{R}^{p}$. The image of an element $k \in \mathbb{N}$ under $s$ is denoted by $s_{k}$ and is called the coefficient of $k$ in $s$. A formal power series $s$ is typically represented by a formal infinite sum of the form

$$
s=\sum_{k \in \mathbb{N}} s_{k} z^{-k} .
$$

The set of formal power series in one indeterminate with coefficients in $\mathbb{R}^{p}$ is denoted by $\mathbb{R}^{p}\left[\left[z^{-1}\right]\right]$. The support of a series $s \in \mathbb{R}^{p}\left[\left[z^{-1}\right]\right]$ is defined as $\operatorname{supp}(s)=\left\{k \in \mathbb{N}: s_{k} \neq 0\right\}$, i.e. as the subset of elements of $\mathbb{N}$ the coefficient in $s$ of which is non-zero. A polynomial is a formal power series with finite support. The set of polynomials with coefficients in $\mathbb{R}^{p}$ is denoted by $\mathbb{R}^{p}\left[z^{-1}\right]$.

Formal power series can be combined using various operations. If $k \in \mathbb{N}, \alpha \in \mathbb{R}$ and $r, s \in \mathbb{R}\left[\left[z^{-1}\right]\right]$, then one defines
- the sum of $r$ and $s$, denoted by $r+s$, as

$$
(r+s)_{k}=r_{k}+s_{k},
$$

- the scalar product of $\alpha$ and $r$, denoted by $\alpha s$, as

$$
(\alpha s)_{k}=\alpha s_{k},
$$

- and the Hadamard product of $r$ and $s$, denoted by $r \odot s$, as

$$
(r \odot s)_{k}=r_{k} s_{k} .
$$

The operations defined above are extended to elements of $\mathbb{R}^{p}\left[\left[z^{-1}\right]\right]$ in a component-wise fashion. With scalar product and sum, the sets $\mathbb{R}\left[z^{-1}\right]$ and $\mathbb{R}^{p}\left[\left[z^{-1}\right]\right]$ assume the structure of vector spaces over the field $\mathbb{R}$.

The shift operator $\sigma: \mathbb{R}^{p}\left[\left[z^{-1}\right]\right] \rightarrow \mathbb{R}^{p}\left[\left[z^{-1}\right]\right]$, defined by the property $[\sigma(s)]_{j}=s_{j+1}$ for all $j \in \mathbb{N}$ and for all $s \in \mathbb{R}^{p}\left[\left[z^{-1}\right]\right]$, is an endomorphism on $\mathbb{R}^{p}\left[\left[z^{-1}\right]\right]$ which acts as a "backward shift" on the coefficients of a given formal power series. For notational convenience, we also define the operator $\sigma_{i}: \mathbb{R}^{p}\left[\left[z^{-1}\right]\right] \rightarrow \mathbb{R}^{p}\left[\left[z^{-1}\right]\right]$ by setting $\left[\sigma_{i}(s)\right]_{j}=s_{i+j}$, for all $i, j \in \mathbb{N}$ and for all $s \in \mathbb{R}^{p}\left[\left[z^{-1}\right]\right]$.

\section{B. Some concepts from nonlinear control theory}

The observation space $\mathcal{O}$ of system (1) is the smallest linear space over $\mathbb{R}$ of functions on $X$ containing the functions $h_{1}(\cdot), \ldots, h_{p}(\cdot)$ closed under Lie derivation with respect to the vector field $f(\cdot)$, with $h_{i}(\cdot)$ the $i$-th component of the function $h(\cdot)$. The observation space $\mathcal{O}$ defines the observability codistribution

$$
d \mathcal{O}(x)=\operatorname{span}\{d \psi(x), \psi(x) \in \mathcal{O}\}, x \in X,
$$

in which $d \psi(\cdot)$ denotes the differential of the function $\psi(\cdot)$. The system (1) is said to satisfy the observability rank condition at $x \in X$ if $\operatorname{dim} d \mathcal{O}(x)=n$.

Under the stated assumptions, the Hartman-Grobman theorem ( $c f .[49,4.14])$ guarantees the existence of an homeomorphism $\tau: N \rightarrow M$ of neighborhoods of the origin such that the flows of system (1) and system (2) are locally conjugated by $\tau(\cdot)$, i.e.

$$
\tau \circ \phi_{t}^{f}(x)=e^{A t} \tau(x), \quad x \in N, \quad t \in \mathbb{R}_{+},
$$

provided both sides of the conjugacy equation are defined. This implies that for every $x \in N$ the relation

$$
\frac{\partial \tau}{\partial x} f(x)=A \tau(x),
$$

holds. To ensure that $\tau(\cdot)$ is actually a diffeomorphism and equation

$$
h(x)=C \tau(x),
$$

is also satisfied for every $x \in N$, we make the following assumption.

Assumption 3. The observation space $\mathcal{O}$ of system (1) has finite dimension. 


\section{Facts from sampled-data control theory}

A popular paradigm used to describe mathematically the interplay between analog (i.e. continuous-time) systems and digital (i.e. discrete-time) simulation, control and identification methods is based on sampled-data models $[48,50]$. This paradigm envisages the acquisition of an analog signal, the output of a continuous-time system, and the conversion of such signal into a discrete-time sequence via a sampling operation. The evolution of the sampled continuous-time system, which may or may not be subject to an exogenous input, is then described exactly at every sampling instant through its sampled-data representation.

Let $\left\{t_{k}\right\}_{k \in \mathbb{N}}$ be a given sequence of distinct real numbers, referred to as sampling instants, such that $\lim _{k \rightarrow \pm \infty} t_{k}= \pm \infty$. In applications, often the sampling instants are equally spaced in time. This motivates the following assumption.

Assumption 4. The sampling instants $\left\{t_{k}\right\}_{k \in \mathbb{N}}$ are equidistant, i.e.

$$
t_{k}=k \delta, \quad k \in \mathbb{N},
$$

for some real number $\delta>0$.

The real number $\delta>0$ is referred to as the sampling period. Without loss of generality, it is assumed to be a free parameter that can be assigned.

The sampling operation is modeled by an operator which associates every function to a formal power series the coefficients of which coincide with the value of the function at the sampling instants, i.e. of the form

$$
y: \mathbb{R}_{+} \rightarrow \mathbb{R}^{p} \mapsto \sum_{k \in \mathbb{R}} y\left(t_{k}\right) z^{-k} .
$$

Definition 1. [39] A discrete-time, autonomous, nonlinear system described by the equations

$$
x_{k+1}=\varphi\left(x_{k}\right), \quad y_{k}=\gamma\left(x_{k}\right),
$$

is a sampled-data representation of system (2) if there exists a real number $\delta>0$ such that $\varphi(x)=\phi_{\delta}^{f}(x)$ and $\gamma(x)=h(x)$ for all $x \in X$.

Note that the sampling period $\delta$ parameterizes the nonlinear difference equation which describes the system (9) and, hence, its system-theoretic properties.

Remark 4. The properties of a system and those of its sampled-data representation can be related (see, e.g., in $[39,50-55])$. In particular, if the continuous-time system (1) satisfies the observability rank condition at $x \in X$, then the discrete-time system (77) satisfies the observability rank condition at $x[52]$, i.e.

$$
\operatorname{rank}\left[\begin{array}{c}
\gamma(x) \\
\gamma \circ \varphi(x) \\
\vdots \\
\gamma \circ \varphi^{n-1}(x)
\end{array}\right]=n,
$$

for generic values of $\delta \in(-r, r)$, with $r>0$ the radius of convergence of $h \circ \phi_{t}^{f}(\cdot)$ as a function of $t$ [55].
Remark 5. The sampled-data representation of a linear system described by equations (2) has the form

$$
x_{k+1}=\Phi x_{k}, \quad y_{k}=\Gamma x_{k},
$$

with $\Phi=e^{A \delta}$ and $\Gamma=C$ for some real number $\delta>0 . \Delta$

\section{Preliminary Results}

The solution of the nonlinear identification problem systems of the form (1) requires additional mathematical machinery. Most of the material presented below is borrowed from $[38,56]$ and adapted to the purposes of this work.

\section{A. The notion of realization}

In analogy with the terminology used in nonlinear realization theory [38], we introduce the following definitions.

Definition 2. A formal power series $s \in \mathbb{R}^{p}\left[\left[z^{-1}\right]\right]$ is said to be convergent if there exist scalars $C>0$ and $r>0$ such that

$$
\left|s_{k}\right| \leq C r^{k}, \quad k \in \mathbb{N} .
$$

If $s \in \mathbb{R}^{p}\left[\left[z^{-1}\right]\right]$ satisfies $[10$, then

$$
z \in \mathbb{C} \mapsto \sum_{k \in \mathbb{N}} s_{k} z^{-k},
$$

defines an analytic function on $\left\{z \in \mathbb{C}:|z|>\frac{1}{r}\right\}$ [57].

Lemma 2. Consider the system (1) and let $x(0)=x_{0} \in X$ be the initial condition. If $s \in \mathbb{R}^{p}\left[\left[z^{-1}\right]\right]$ is a formal power series of the form

$$
s_{k}=h \circ \phi_{t_{k}}^{f}\left(x_{0}\right), \quad k \in \mathbb{N},
$$

with $\delta>0$ and $\left\{t_{k}\right\}_{k \in \mathbb{N}}$ as in (6), then $s$ is convergent.

Proof. The claim is a direct consequence of Assumption 2

Definition 3. A formal power series $s \in \mathbb{R}^{p}\left[\left[z^{-1}\right]\right]$ of the form (11) is called a generating series for the system (1).

Definition 4. Let $\delta>0$ and $\left\{t_{k}\right\}_{k \in \mathbb{N}}$ be as in (6). A system of the form (1) is called a realization of the convergent formal power series $s \in \mathbb{R}^{p}\left[\left[z^{-1}\right]\right]$ around $x_{0} \in \mathbb{R}^{n}$ corresponding to $\left\{t_{k}\right\}_{k \in \mathbb{N}}$ if the vector field $f(\cdot)$ and the function $h(\cdot)$ are defined in a neighborhood of $x_{0}$ and satisfy the condition 11.

Note that the notion of realization introduced above is fundamentally different from the classical one $[38,58]$. Nonlinear realization theory uses the fact that the observation space at a point completely specifies the output of an analytic system to define the notion of realization. On the other hand, Definition 4 is based on the correspondence between the output of a system of the form (1) and its values at equidistant sampling instants, which, in general, is not one-to-one. This raises a question of identifiability.

Remark 6. For a linear system described by equations of the form (2), a generating series assumes necessarily the form

$$
s_{k}=C e^{A t_{k}} x_{0}, \quad k \in \mathbb{N},
$$

with $\delta>0,\left\{t_{k}\right\}_{k \in \mathbb{N}}$ as in [6], and $x(0)=x_{0} \in \mathbb{R}^{n}$ the initial condition of the state of the system. 


\section{B. The Hankel mapping}

Inspired and motivated by the mathematical theories of linear and nonlinear realization $[38,58]$, we now introduce the following notions and assumptions.

\section{Definition 5. The Hankel mapping}

$$
\mathcal{H}_{s}: \mathbb{R}\left[z^{-1}\right] \rightarrow \mathbb{R}^{p}\left[\left[z^{-1}\right]\right]
$$

associated with a convergent formal power series $s \in \mathbb{R}^{p}\left[\left[z^{-1}\right]\right]$ is the morphism of real vector spaces uniquely specified by the property $\left[\mathcal{H}_{s}\left(z^{-i}\right)\right]_{j}=s_{i+j}$ for all $i, j \in \mathbb{N}$.

Definition 6. A convergent formal power series $s \in \mathbb{R}\left[\left[z^{-1}\right]\right]$ is said to be admissible if there exist polynomials $p_{1}, \ldots, p_{n} \in \mathbb{R}\left[z^{-1}\right]$ which generate a subspace $V$ such that

$$
\operatorname{Im} \mathcal{H}_{s}=\left\{\sigma_{i}\left(H_{s}(p)\right): i \in \mathbb{N}, p \in V\right\} .
$$

Definition 7. The Hankel rank $\operatorname{rank}_{\mathrm{H}}(s)$ of an admissible formal power series $s \in \mathbb{R}^{p}\left[\left[z^{-1}\right]\right]$ is defined as $\operatorname{rank}_{\mathrm{H}}(s)=\operatorname{dim} \mathcal{H}_{s}\left(\mathbb{R}\left[z^{-1}\right]\right)$, i.e. as the dimension of the image under $\mathcal{H}_{s}$ of the whole space $\mathbb{R}\left[z^{-1}\right]$.

Definition 8. The Lie rank $\operatorname{rank}_{\mathrm{L}}(s)$ of an admissible formal power series $s \in \mathbb{R}^{p}\left[\left[z^{-1}\right]\right]$ is defined as $\operatorname{rank}_{\mathrm{L}}(s)=\operatorname{dim} \mathcal{H}_{s}(V)$, i.e. as the dimension of the image of the restriction of $\mathcal{H}_{s}$ to the subspace $V$.

Representing the elements of the vector spaces $\mathbb{R}\left[z^{-1}\right]$ and $\mathbb{R}^{p}\left[\left[z^{-1}\right]\right]$ as real column vectors, the infinitely many entries of which are real numbers and real vectors with $p$ components indexed by elements of $\mathbb{N}$, it is possible to derive a matrix representation of the linear transformation $\mathcal{H}_{s}$. In particular, such representation has the form

$$
H_{s}=\left[\begin{array}{cccc}
s_{0} & s_{1} & s_{2} & \cdots \\
s_{1} & s_{2} & s_{3} & \cdots \\
s_{2} & s_{3} & s_{4} & \cdots \\
\vdots & \vdots & \vdots & \ddots
\end{array}\right] .
$$

The infinite matrix $H_{s}$ is referred to as the Hankel matrix of the series $s$. The rank ${ }^{3}$ of the Hankel matrix $H_{s}$ coincides, by definition, with the Hankel rank of $s$, i.e. $\operatorname{rank}_{\mathrm{H}}(s)=$ $\operatorname{rank} H_{s}$.

As one would expect, the matrix representation of the Hankel mapping associated to the generating series produced by a linear system admits a special decomposition.

Remark 7. Consider a generating series $s \in \mathbb{R}^{p}\left[\left[z^{-1}\right]\right]$ associated with a linear system of the form (2), i.e. such that $s_{k}=C e^{A t_{k}} x_{0}$ for all $k \in \mathbb{N}$, with $x(0)=x_{0} \in \mathbb{R}^{n}$ the initial condition of the state. The Hankel matrix associated with the given formal power series $s$, by Assumption 4 has the form $H_{s}=O_{s} R_{s}$, in which $O_{s}=\left[C^{\prime} e^{A^{\prime} t_{1}} C^{\prime} e^{A^{\prime} t_{2}} C^{\prime} \cdots\right]^{\prime}$ and $R_{s}=\left[\begin{array}{lll}x_{0} & \left.e^{A t_{1}} x_{0} e^{A t_{2}} x_{0} \cdots\right]\end{array}\right]$

\footnotetext{
${ }^{3}$ The rank of a matrix (even an infinite one) can be defined as the dimension of its largest non-vanishing sub-determinant [59]. With such a definition, the rank of a matrix coincides with the dimension of the space generated by its rows (and by its columns).
}

\section{MAIN RESULTS}

To streamline the presentation of our main results, given an admissible formal power series $s \in \mathbb{R}^{p}\left[\left[z^{-1}\right]\right]$ and two sets

$$
\begin{aligned}
K & =\left\{k_{1}, \ldots, k_{n}: k_{i} \in \mathbb{N}, k_{i}<k_{j}, i<j\right\}, \\
L & =\left\{l_{1}, \ldots, l_{n}: l_{i} \in \mathbb{N}, l_{i}<l_{j}, i<j\right\},
\end{aligned}
$$

define the square matrices

$$
H_{s}^{K, L}=\left[\begin{array}{cccc}
s_{k_{1}+l_{1}} & s_{k_{1}+l_{2}} & \cdots & s_{k_{1}+l_{n}} \\
s_{k_{2}+l_{1}} & s_{k_{2}+l_{2}} & \cdots & s_{k_{2}+l_{n}} \\
\vdots & \vdots & \ddots & \vdots \\
s_{k_{n}+l_{1}} & s_{k_{n}+l_{2}} & \cdots & s_{k_{n}+l_{n}}
\end{array}\right]
$$

and

$$
\sigma H_{s}^{K, L}=\left[\begin{array}{cccc}
s_{k_{2}+l_{1}+1} & s_{k_{2}+l_{2}+1} & \cdots & s_{k_{2}+l_{n}+1} \\
s_{k_{3}+l_{1}} & s_{k_{3}+l_{2}+1} & \cdots & s_{k_{3}+l_{n}+1} \\
\vdots & \vdots & \ddots & \vdots \\
s_{k_{n}+l_{1}+1} & s_{k_{n}+l_{2}+1} & \cdots & s_{k_{n}+l_{n}+1}
\end{array}\right] .
$$

\section{A. Linear systems}

The main result of this part concerns the existence of linear realizations of a convergent formal power series.

Theorem 1. Let $s \in \mathbb{R}^{p}\left[\left[z^{-1}\right]\right]$ be an admissible formal power series and let $\delta>0$ be the sampling period. Consider the following statements.

L1) The Hankel rank of $s$ is finite, i.e. $\operatorname{rank}_{\mathrm{H}}(s)=n<\infty$. Moreover, given two sets $K$ and $L$ of the form (12), if $\Pi=H_{s}^{K, L}$ is full rank, then $\sigma \Pi=\sigma H_{s}^{K, L}$ is also full rank and the unique solution of the matrix equation

$$
\Pi S=\sigma \Pi
$$

is such that $\rho(S)<1$.

L2) There exists a realization of $s$ of the form (2) and the sampled-data representation (9) with sampling period $\delta$ is such that the pair $(\Phi, \Gamma)$ is observable. Then L1) $\Rightarrow$ L2)

Remark 8. The claim can be proved using a modification of Silverman's algorithm [60]. For convenience of the reader familiar with that kind of proof, the exposition closely follows that of [58, Lemma 4.41]. The proof is given to draw a parallel with the seemingly different argument used for nonlinear systems.

Proof. By hypothesis, there exist $n=\operatorname{rank}_{\mathrm{H}}(s)$ not necessarily consecutive columns of $H_{s}$ that span its column space. Let $\Pi$ be the matrix formed by these columns and, by a convenient abuse of notation, let $\sigma \Pi$ be the matrix obtained by shifting those of $\Pi$ by one column. In addition, Let $P$ be the first column of $H_{s}$. Note that the equations

$$
\begin{aligned}
\Pi e^{\bar{A} \delta} & =\sigma \Pi, \\
\Pi \chi & =P,
\end{aligned}
$$

uniquely specify a non-singular matrix $\bar{A}$ and a vector $\chi$, as the columns of $\Pi$ form a basis for the space spanned by the 
columns of $H_{s}$ and, by assumption, $\sigma \Pi$ is full rank. Lastly, define $\bar{C}$ as the matrix formed by the first $p$ rows of $\Pi$.

Observe that (13) imply the identity

$$
(P)_{1}=(\Pi \bar{x})_{1}=(\Pi)_{1} \bar{x}=\bar{C} \bar{x},
$$

in which $(P)_{k}, k>0$, denotes the $k$-th block row with $p$ components of the matrix $P$. More generally, letting $\bar{S}=e^{\bar{A} \delta}$ and denoting by $\sigma^{k} \Pi$ the shift by $k$ columns of $\Pi$ (with some abuse of notation once again) yields

$$
\left(\sigma^{k} P\right)_{1}=\left(\sigma^{k} \Pi \chi\right)_{1}=\left(\Pi \bar{S}^{k} \chi\right)_{1}=(\Pi)_{1} \bar{S}^{k} \chi=\bar{C} \bar{S}^{k} \chi,
$$

for all $k>0$, thus proving that the pair $(\bar{A}, \bar{C})$ defines a realization of $s$ at $\chi$ of dimension $n$. The rest of the proof follows, by construction, from the definition of sampled-data representation and hence is omitted.

Remark 9. In anticipation of the analysis of the nonlinear case, it is worth noting that for a linear system of the form (2) the Schröder equation boils down to a conjugacy equation

$$
\bar{\Psi} \circ \bar{S} \chi=\bar{S} \bar{\Psi}(\chi), \quad \chi \in M=\mathbb{R}^{n},
$$

which admits a unique, trivial, solution linear in its argument and fulfilling the condition

$$
\left.\frac{\partial \bar{\Psi}}{\partial \chi}\right|_{\chi=0}=I
$$

i.e. the identity function. Thus, using the approach outlined in Section II] it follows that the the flow of the system reads as

$$
t \mapsto \bar{S}^{\frac{t}{\delta}} \chi, \quad \chi \in M,
$$

and the corresponding vector field is given by

$$
\left.\frac{d}{d t} \bar{S}^{\frac{t}{\delta}}\right|_{t=0}=\bar{A} \chi, \quad \chi \in M
$$

with $\bar{A}=T A T^{-1}$ and $T$ a non-singular square matrix. $\triangle$

\section{B. Nonlinear systems}

We now focus on the identification problem for systems of the form (1). To this end, the problem is reformulated as follows.

Given an admissible formal power series $s \in \mathbb{R}^{p}\left[\left[z^{-1}\right]\right]$, and a set of polynomials $p_{1}, \ldots, p_{n} \in V$, which, without loss of generality, can be assumed to be monomials ${ }^{4}$ i.e. such that $p_{i}=z^{l_{i}}$, with $l_{i} \in L$, for all $i \in[1, n]$ and for some set $L$ of the form (12b), define the formal power series

$$
r(\chi)=\sum_{k=0}^{\infty} \frac{1}{k !}\left(\sum_{i=1}^{n} \chi_{i} p_{i}\right)^{k},
$$

parameterized by the real vector $\chi=\left(\chi_{1}, \ldots, \chi_{n}\right) \in \mathbb{R}^{n}$, and the real-valued analytic functions

$$
h_{i}(\chi)=\sum_{k=0}^{\infty}\left[\sigma_{i}(s) \odot r(\chi)\right]_{k}, \quad i \in \mathbb{N} .
$$

\footnotetext{
${ }^{4}$ The same conclusions hold when $p_{1}, \ldots, p_{n}$ are polynomials by linearity of the map $\mathcal{H}_{s}$.
}

Note that, for every fixed $\chi \in \mathbb{R}^{n}, h_{i}(\chi)$ is given by the sum of a numerical series, the convergence of which is guaranteed around the origin by $s$ being a convergent formal power series. Every function $h_{i}(\cdot), i \in \mathbb{N}$, is thus well-defined in a neighborhood of the origin.

With these definitions, the problem of determining a realization of $s$ amounts to finding a vector field $\theta(\cdot)$ defined in a neighborhood $M$ of the origin such that

$$
h_{i} \circ \bar{\varphi}(\chi)=h_{i+1}(\chi), \quad \chi \in M, \quad i \in \mathbb{N},
$$

in which $\delta>0$ is the sampling period and $\bar{\varphi}(\chi)=\phi_{\delta}^{\theta}(\chi)$ for all $\chi \in M$. The property (16) implies in fact that the function $h_{0}(\cdot)$ and the vector field $\theta(\cdot)$ constitute a realization of $s$ at the origin of $\mathbb{R}^{n}$, since $h_{i}(0)=s_{i}$ for all $i \in \mathbb{N}$.

Before stating and proving the main result, a preliminary lemma is stated.

Lemma 3. [40, Lemma 6.1] Let $\bar{\varphi}(\cdot)$ be a function defined on a set $M$ such that $\bar{\varphi}(M) \subseteq M$, let $\tau(\cdot)$ be a one-to-one mapping of $M$ onto a set $N$ and let

$$
\varphi(x)=\tau \circ \bar{\varphi} \circ \tau^{-1}(x), \quad x \in N .
$$

If $\Psi(\cdot)$ solves $\Psi \circ \varphi(x)=S \Psi(x)$ on $N$, then

$$
\bar{\Psi}(\chi)=\Psi \circ \tau(\chi), \quad \chi \in M,
$$

satisfies the equation $\bar{\Psi} \circ \bar{\varphi}(\chi)=S \bar{\Psi}(\chi)$ on $M$.

Theorem 2. Let $s \in \mathbb{R}^{p}\left[\left[z^{-1}\right]\right]$ be a convergent formal power series and let $\delta>0$ be the sampling period. Consider the following statements.

NL1) The Lie rank of $s$ is finite, i.e. $\operatorname{rank}_{\mathrm{L}}(s)=n<\infty$. Moreover, given two sets $K$ and $L$ of the form (12) such that $p_{i}=z^{l_{i}} \in V$ for all $i \in[1, n]$, if $\Pi=H_{s}^{K, L}$ is full rank, then $\sigma \Pi=\sigma H_{s}^{K, L}$ is also full rank and the unique solution of the matrix equation

$$
\Pi S=\sigma \Pi
$$

is such that $\rho(S)<1$.

NL2) There exists a realization of $s$ of the form (2) defined on a neighborhood $M$ of the origin and the sampled-data representation (9) with sampling period $\delta$ is such that the condition (8) is satisfied at the origin.

Then NL1) $\Rightarrow$ NL2)

Proof. The proof of this implication is logically organized as follows.

(i) First, a diffeomorphism $\bar{\varphi}(\cdot)$ locally defined in a neighborhood $M$ of the origin is constructed in a way that ensures the compatibility with the conditions in 16 .

(ii) Then, the iterates of $\bar{\varphi}(\cdot)$ are interpolated using the solution of the Schröder equation (5), thus yielding a map $\bar{\varphi}_{t}: M \rightarrow M$ defined for all $t \in \mathbb{R}_{+}$.

(iii) Finally, a vector field $\theta(\cdot)$ is defined via the formula

$$
\theta(\chi)=\left.\frac{d}{d t} \bar{\varphi}_{t}(\chi)\right|_{t=0}, \quad \chi \in M .
$$


The reader familiar with nonlinear realization theory should notice that part (i) is achieved using an argument similar to one presented in the proof of [38, Theorem 3.4.4]. To simplify the exposition, as in [38, Theorem 3.4.4], the claim is proved only for the single-output case, i.e. $p=1$.

(i). By hypothesis, there exist polynomials $p_{1}, \ldots, p_{n} \in V$ such that the formal power series $\mathcal{H}_{s}\left(p_{1}\right), \ldots, \mathcal{H}_{s}\left(p_{n}\right)$ form a basis for the space spanned by the columns of $H_{s}$. Using such polynomials, define the formal power series (14) and (15).

The linear independence of the formal power series $\mathcal{H}_{s}\left(p_{1}\right), \ldots, \mathcal{H}_{s}\left(p_{n}\right)$ over the field $\mathbb{R}$ implies the existence of $n$ integers $k_{1}, \ldots, k_{n} \in \mathbb{N}$ such that

$$
\operatorname{rank}\left[\begin{array}{ccc}
{\left[\mathcal{H}_{s}\left(p_{1}\right)\right]_{k_{1}}} & \cdots & {\left[\mathcal{H}_{s}\left(p_{n}\right)\right]_{k_{1}}} \\
\vdots & \ddots & \vdots \\
{\left[\mathcal{H}_{s}\left(p_{1}\right)\right]_{k_{n}}} & \cdots & {\left[\mathcal{H}_{s}\left(p_{n}\right)\right]_{k_{n}}}
\end{array}\right]=n .
$$

Then

$$
\left[\mathcal{H}_{s}\left(p_{i}\right)\right]_{k_{j}}=s_{k_{j}+l_{i}}=\left[\sigma_{k_{j}}(s)\right]_{l_{i}}=\left.\frac{\partial h_{k_{j}}}{\partial \chi}\right|_{\chi=0},
$$

where the second and the third equalities follow from the definition of the mappings $\mathcal{H}_{s}$ and $\sigma_{k_{j}}$.

As a consequence, the mappings

$$
\chi \mapsto \pi(\chi)=\left[\begin{array}{lll}
h_{k_{1}}(\chi) & \cdots & h_{k_{n}}(\chi)
\end{array}\right]^{\prime},
$$

and

$$
\chi \mapsto \sigma \pi(\chi)=\left[\begin{array}{lll}
h_{k_{2}}(\chi) & \cdots & h_{k_{n}+1}(\chi)
\end{array}\right]^{\prime},
$$

are full rank at the origin, i.e. the matrices

$$
\Pi=\left.\frac{\partial \pi}{\partial \chi}\right|_{\chi=0}
$$

and

$$
\sigma \Pi=\left.\frac{\partial \sigma \pi}{\partial \chi}\right|_{\chi=0}
$$

are non-singular.

Fix $\chi \in \mathbb{R}^{n}$ and consider the equation

$$
\pi(\bar{\varphi})=\sigma \pi(\chi)
$$

in the unknown vector $\bar{\varphi} \in \mathbb{R}^{n}$. Note that the matrix of partial derivatives of $\pi(\cdot)(\sigma \pi(\cdot)$, resp.) is non-singular in a neighborhood $M$ of the origin, since its value is non-singular therein. Thus, the solution of (18) defines a diffeomorphism $\bar{\varphi}: M \rightarrow M$ with the property $h_{k_{j}} \circ \bar{\varphi}(\chi)=h_{k_{j}+1}(\chi)$ for every $j \in[1, n]$. To show that the property $(16)$ is also possessed by all other functions $h_{k}(\cdot)$, with $k \in \mathbb{N}$, it suffices to consider that every formal power series in $\mathcal{H}_{s}(V)$ is a linear combination over $\mathbb{R}$ of shifts of the elements $\mathcal{H}_{s}\left(p_{1}\right), \ldots, \mathcal{H}_{s}\left(p_{n}\right)$

By Lemma 3, it is enough to consider a translation of the origin of the coordinate system to ensure that the condition $\bar{\varphi}(0)=0$ is satisfied.

(ii). Consider now the Schröder equation

$$
\bar{\Psi} \circ \bar{\varphi}(\chi)=\bar{S} \bar{\Psi}(\chi), \quad \chi \in M,
$$

in which the matrix $S$ is uniquely specified by the matrix equation

$$
\Pi=S \sigma \Pi \text {. }
$$

By assumption, $\rho(S)<1$. Thus, in view of [41, Theorem $8.2 .1]$, there is a unique analytic solution $\bar{\Psi}(\cdot)$ in a neighborhood of the origin fulfilling the condition

$$
\left.\frac{\partial \Psi}{\partial \chi}\right|_{\chi=0}=I
$$

Without loss of generality, we may identify such neighborhood with $M$, since similar considerations can be performed on an appropriate restricted neighborhood otherwise.

Define the family of mappings

$$
\bar{\varphi}_{t}=\bar{\Psi}^{-1}\left(\bar{S}^{\frac{t}{\delta}} \bar{\Psi}(\chi)\right), \quad t \in \mathbb{R}_{+}, \quad \chi \in M,
$$

and note that it defines a one-parameter group of diffeomorphisms, since $\bar{\varphi}_{0}(\chi)=\chi$ and $\bar{\varphi}_{t} \circ \bar{\varphi}_{v}=\bar{\varphi}_{v} \circ \bar{\varphi}_{t}$ for all $t, v \in \mathbb{R}_{+}$. (17).

(iii). The vector field $\theta(\cdot)$ is determined using the formula

To complete the proof, note that, by construction, the sampled-data representation with sampling period $\delta$ of the system specified by $h_{0}(\cdot)$ and $\theta(\cdot)$ is such that the condition (8) is satisfied at the origin.

\section{CONCLUSION}

The identification problem for continuous-time autonomous nonlinear systems has been discussed. A framework which combines the use of functional equations with realization-theoretic techniques has been proposed. Under certain conditions, solutions are constructed based on discrete-time output data. Future research should be devoted to the extension of the proposed theoretical framework to systems with inputs and to the study of its practical applications.

\section{REFERENCES}

[1] T. D. Söderström and P. G. Stoica, System identification. Upper Saddle River, NJ: Prentice Hall, 1989.

[2] L. Ljung, System identification - theory for the user (2nd edition). Upper Saddle River, NJ: Prentice Hall, 1999.

[3] B. Huang and R. Kadali, Dynamic modeling, predictive control and performance monitoring: A data-driven subspace approach. New York: Springer-Verlag, 2008.

[4] R. Pintelon and J. Schoukens, System identification: a frequency domain approach (2nd edition). Hoboken, NJ: Wiley-IEEE Press, 2012.

[5] P. Van Overschee and B. De Moor, Subspace identification for linear systems: theory - implementation - applications. Dordrecht, The Netherlands: Kluwer Academic Publishers, 1996.

[6] S. Bittanti and G. Picci, Eds., Identification, adaptation, learning: the science of learning models from data. Berlin, Germany: SpringerVerlag, 1996.

[7] T. Katayama, Subspace methods for system identification. London, U.K.: Springer, 2006.

[8] M. Verhaegen and V. Verdult, Filtering and system identification: a least squares approach. Cambridge, U.K.: Cambridge University Press, 2007.

[9] G. Liu, Nonlinear identification and control: a neural network approach. New York: Springer-Verlag, 2001.

[10] K. Schittkowski, Numerical data fitting in dynamical systems. Dordrecht, The Netherlands: Kluwer Academic Publishers, 2002. 
[11] R. Murray-Smith and T. Johansen, Multiple model approaches to modelling and control. London, U.K.: Taylor \& Francis, 1997.

[12] H. Kantz and T. Schreiber, Nonlinear time series analysis (2nd edition). Cambridge, U.K.: Cambridge University Press, 2004.

[13] L. Ljung, "Perspectives on system identification," Annu. Rev. Control, vol. 34, no. 1, pp. 1-12, 2010.

[14] A. Doucet and V. B. Tadić, "Parameter estimation in general statespace models using particle methods," Ann. Inst. Statist. Math, vol. 55, no. 2, pp. 409-422, 2003.

[15] T. B. Schön, A. Wills, and B. Ninness, "System identification of nonlinear state-space models," Automatica, vol. 47, no. 1, pp. 39-49, 2011.

[16] W. Pan, Y. Yuan, J. Goncalves, and G. B. Stan, "A sparse bayesian approach to the identification of nonlinear state-space systems," IEEE Trans. Autom. Control, 2015, to be published.

[17] D. Westwick and M. Verhaegen, "Identifying MIMO Wiener systems using subspace model identification methods ," Signal Proc., vol. 52, pp. $235-258,1996$.

[18] M. Lovera, T. Gustafsson, and M. Verhaegen, "Recursive subspace identification of linear and non-linear Wiener state-space models," Automatica, vol. 36, no. 11, pp. 1639-1650, 2000.

[19] J. C. Gómez and E. Baeyens, "Subspace identification of multivariable Hammerstein and Wiener models," in Proc. 15th IFAC World Congr., Barcelona, Spain, 2002, pp. 418-418.

[20] I. Goethals, K. Pelckmans, J. A. K. Suykens, and B. De Moor, "Subspace identification of Hammerstein systems using least squares support vector machines," IEEE Trans. Autom. Control, vol. 50, no. 10, pp. 1509-1519, Oct. 2005.

[21] A. Wills, T. B. Schön, L. Ljung, and B. Ninness, "Identification of HammersteinWiener models," Automatica, vol. 49, no. 1, pp. 70 - 81, 2013.

[22] G. Pillonetto, F. Dinuzzo, T. Chen, G. De Nicolao, and L. Ljung, "Kernel methods in system identification, machine learning and function estimation: A survey," Automatica, vol. 50, no. 3, pp. 657-682, 2014.

[23] W. Favoreel, B. De Moor, and P. Van Overschee, "Subspace identification of bilinear systems subject to white inputs," IEEE Trans. Autom. Control, vol. 44, no. 6, pp. 1157-1165, Jun. 1999.

[24] H. Chen and J. M. Maciejowski, "A new subspace identification method for bilinear systems," Dept. Eng., Univ. Cambridge, Cambridge, U.K., Tech. Rep. CUED/F-INFENG/TR.357, 2000.

[25] V. Verdult, M. Verhaegen, and J. Scherpen, "Identification of nonlinear nonautonomous state space systems from input-output measurements," in Proc. IEEE Int. Conf. Ind. Technol., Goa, India, 2000, pp. 410-414.

[26] V. Verdult, "Nonlinear System Identification: A State-Space Approach," Ph.D. dissertation, 2002.

[27] G. Kerschen, K. Worden, A. F. Vakakis, and J. C. Golinval, "Past, present and future of nonlinear system identification in structural dynamics," Mech. Syst. Sig. Process., vol. 20, no. 3, pp. 505-592, 2006.

[28] S. Marchesiello and L. Garibaldi, "A time domain approach for identifying nonlinear vibrating structures by subspace methods," Mech. Syst. Sig. Process., vol. 22, no. 1, pp. 81-101, 2008.

[29] D. S. Broomhead and G. P. King, "Extracting qualitative dynamics from experimental data," Phys. D, vol. 20, no. 2, pp. 217-236, 1986.

[30] M. Casdagli, S. Eubank, J. Doyne Farmer, and J. Gibson, "State-space reconstruction in the presence of noise," Phys. D, vol. 51, no. 1, pp. $52-98,1991$

[31] T. Sauer, J. A. Yorke, and M. Casdagli, "Embedology," J. Stat. Phys., vol. 65, no. 3-4, pp. 579-616, 1991.

[32] J. F. Gibson, J. Doyne Farmer, M. Casdagli, and S. Eubank, "An analytic approach to practical state-space reconstruction," Phys. D, vol. 57, no. 1, pp. 1-30, 1992.

[33] J. Stark, "Delay embeddings for forced systems. I. Deterministic forcing," J. Nonlinear Science, vol. 9, no. 3, pp. 255-332, 1999

[34] J. Stark, D. S. Broomhead, M. E. Davies, and J. Huke, "Delay embeddings for forced systems. II. Stochastic forcing," J. Nonlinear Science, vol. 13, no. 6, pp. 519-577, 2003.

[35] N. H. Packard, J. P. Crutchfield, J. D. Farmer, and R. S. Shaw, "Geometry from a time series," Phys. Rev. Lett., vol. 45, no. 9, pp. $712-715,1980$.

[36] F. Takens, "Detecting strange attractors in turbulence," in Lecture Notes in Mathematics. Berlin, Germany: Springer-Verlag, 1981, vol. 898, pp. 366-381.

[37] A. Mees, P. Rapp, and L. Jennings, "Singular-value decomposition and embedding dimension," Phys. Rev. A, vol. 36, no. 1, p. 340, 1987.
[38] A. Isidori, Nonlinear control systems (3rd edition). New York: Springer-Verlag, 1995.

[39] H. Nijmeijer and A. Van der Schaft, Nonlinear dynamical control systems. New York: Springer-Verlag, 1990.

[40] M. Kuczma, Functional equations in a single variable. Warszawa, Poland: Polish Scientific, 1968.

[41] M. Kuczma, B. Choczewski, and R. Ger, Iterative functional equations. Cambridge, U.K.: Cambridge University Press, 1990.

[42] R. D. Enoch, "Formal power series solutions of Schröder's equation," Aequationes mathematicae, vol. 74, no. 1-2, pp. 26-61, 2007.

[43] P. Hartman, "Ordinary differential equations," New York, 1964.

[44] H. K. Khalil, Nonlinear systems (3rd edition). Upper Saddle River, N.J.: Prentice Hall, 1996.

[45] A. Padoan and A. Astolfi, "Dimension estimation for autonomous nonlinear systems," Proc. 54th Conf. Decision Control, Osaka, Japan, 2015, to be published.

[46] C. Reutenauer, Free Lie algebras. Oxford, U.K.: Clarendon Press, 1993.

[47] J. Berstel and C. Reutenauer, Noncommutative rational series with applications. Cambridge, U.K.: Cambridge University Press, 2011.

[48] J. I. Yuz and G. C. Goodwin, Sampled-data models for linear and nonlinear systems. London, U. K.: Springer-Verlag, 2014.

[49] C. Chicone, Ordinary differential equations with applications (2nd edition). New York: Springer, 2006.

[50] E. D. Sontag, Mathematical control theory: deterministic finite dimensional systems (2nd edition). New York: Springer-Verlag, 1998.

[51] R. E. Kalman, Y. C. Ho, and K. S. Narendra, "Controllability of linear dynamical systems," Contr. Diff. Eq., vol. 1, no. 2, pp. 189-213, 1962.

[52] H. Nijmeijer, "Observability of autonomous discrete time non-linear systems: a geometric approach," Int. J. Control, vol. 36, no. 5, pp. 867-874, 1982.

[53] F. Albertini and E. D. Sontag, "Discrete-time transitivity and accessibility: analytic systems," SIAM J. Control Optim., vol. 31, no. 6, pp. 1599-1622, 1993.

[54] S. Ammar and J. C. Vivalda, "On the preservation of observability under sampling," Syst. Control Lett., vol. 52, no. 1, pp. 7-15, 2004.

[55] A. Padoan and A. Astolfi, "A note on delay coordinates for locally observable analytic systems," IEEE Trans. Autom. Control, to be published.

[56] Y. Wang and E. D. Sontag, "Generating series and nonlinear systems: analytic aspects, local realizability, and i/o representations," in Forum Mathematicum, vol. 4, no. 3, 1992, pp. 299-322.

[57] W. Rudin, Real and complex analysis (2nd edition). New York: McGraw-Hill, 1974.

[58] A. C. Antoulas, Approximation of large-scale dynamical systems. Philadelphia, PA: SIAM, 2005.

[59] F. Gantmacher, The theory of matrices. New York: Chelsea, 1959, vol. II.

[60] L. M. Silverman, "Structural properties of time-variable linear systems," Ph.D. dissertation, Dept. Elect. Eng., Columbia Univ., New York, 1966 